Journal of Accident and Emergency Medicine 1995 12, 206-207

\title{
The continuing need to formalize pre-hospital triage, highlighted by a case of unusual injury
}

\author{
M. LAVIS, S. MCCARTHY \& N. H. JENKINS
}

Accident and Emergency Department, Nevill Hall and District NHS Trust, Abergavenny, Gwent, UK

\section{SUMMARY}

A case is reported of portal vein tear, which, although ultimately fatal, as a result of massive haemorrhage, demonstrates the continuing need to formalize and audit pre-hospital tirage.

Key words: portal vein, pre-hospital triage

\section{INTRODUCTION}

Injury involving the portal vein following blunt abdominal trauma is unusual. The case presented serves to raise the question of appropriate transfer of injured patients by paramedics, the importance of adherence to Advanced Cardiac Life Support (ACLS) and Advanced Trauma Life Support (ATLS) protocols, ${ }^{1,2}$ and the safe use of seat belts.

\section{CASE REPORT}

A 46-year-old woman was the front-seat passenger of a van involved in a traffic accident during which the vehicle had collided with the road verge. She was wearing a 3-point static seat belt. The scene was attended by a Paramedic crew after $19 \mathrm{~min}$, when her blood pressure was $80 / 40 \mathrm{mmHg}$, pulse rate was 100 , respiratory rate was 14 and her Glasgow Coma Scale (G.C.S.) was 13/15, giving a Revised Trauma Score of 11/12. Following the application of a cervical collar, provision of oxygen and administration of $2.5 \mathrm{~L}$ of fluid $(2 \mathrm{~L}$ of colloid and $0.5 \mathrm{~L}$ of crystalloid) she was transported to the local general practitioner (GP lead hospital.

Correspondence: Mark Lavis, Staff Doctor in Accident and Emergency Medicine, Nevill Hall and District NHS Trust, Abergavenny, Gwent NP7 7EG, UK
Following a further deterioration of vital signs she was then transported to the local) District General Hospital (DGH), receiving an additional $2 \mathrm{~L}$ of colloid.

On arrival in the accident and emergency (A\&E) department, $128 \mathrm{~min}$ after the accident, the patient had been in a state of cardiorespiratory arrest for 2 min, during which time she had received Basic
Life Support. Advanced Life Support was instituted by way of endotracheal intubation with in-line stabilization of the cervical spine and subsequent ventilation with $100 \%$ oxygen. Cardiac monitoring at this stage revealed a sinus tachycardia of $130 \mathrm{~min}^{-1}$, but the patient's pulse remained impalpable. Clinical examination revealed no obvious intra-thoracic injury, but developing distension and bruising of the abdomen.

A diagnosis of electromechanical dissociation cardiac arrest secondary to hypovolaemia was made and treated with a total of $4 \mathrm{~L}$ of whole blood and $4 \mathrm{~L}$ of colloid. Throughout this period the cardiac arrest per se was 'managed' by a senior house officer (SHO) in A\&E accredited in ACLS.' The patient received ongoing cardiopulmonary resuscitation and a total of 24 separate 1-mg doses of adrenaline. The trauma element was handled by an A\&E SHO, a staff grade doctor and a consultant, each of whom was accredited in ATLS. ${ }^{2}$ During the resuscitation a variable spontaneous cardiac output was achieved, and the patient responded by opening her eyes.

The patient underwent laparotomy $25 \mathrm{~min}$ following arrival at the DGH, but died during the procedure. Subsequent post-mortem examination revealed extensive intraperitoneal haemorrhage following a torn portal vein and avulsion of the common bile duct. There were associated fractures of the right sixth to tenth ribs. There was no other significant injury.

\section{DISCUSSION}

Injury to the portal vein is rare and usually fatal. A total of 21 patients with extra hepatic injuries to the portal triad (site of injury not specified), representing $0.21 \%$ of all patients admitted with multiple trauma over an 11-year period, were reviewed. Of these, 14 patients had a portal vein injury, while the remainder sustained injury to the 
Pre-hospital triage: portail vein injury hepatic artery, the common bile duct or a combination of these injuries. The majority of these patients died from uncontrolled haemorrhage. ${ }^{3}$

A further study ${ }^{4}$ noted that injuries involving the portal vein invariably involved complete pancreatic rupture and, in the absence of such pancreatic trauma, the case reported here is unusual.

We postulate that the 3-point static seat belt used by this patient was probably the cause of her injury, and this has important implications in the area of accident prevention.

This case illustrates the need to formalize and implement effective pre-hospital triage. This patient had an initial Revised Trauma Score (RTS) of 11/12, a score associated with a mortality of at least $10 \%$, and was indirectly transferred to the nearest A\&E department in excess of $2 \mathrm{~h}$ posttrauma, in a state of cardiorespiratory arrest secondary to hypovolaemia. Many Paramedic crews routinely record the RTS, but the noting of such a score is meaningless unless appropriate action is taken. When RTS scoring is used, depending on locally agreed policies, patients scoring poorly should be transferred directly to the nearest A\&E department, rather than to facilities offering less specialized care.

The effectiveness of a resuscitation team, adhering to ACLS ${ }^{1}$ and ATLS $^{2}$ protocols, is illustrated by the temporary return of a spontaneous cardiac output and neurological function, despite massive haemorrhage and the delay before initiating advanced life support measures. In a similarly physiologically compromised patient with a more surgically amenable injury the outcome may well have been favourable.

The continuing need for training, clear guidelines and audit with respect to pre-hospital triage is evident.

\section{ACKNOWLEDGEMENTS}

The author wishes to thank Mrs Ann Rees and Mrs Mari C. Finney for their assistance with the preparation of this manuscript.

\section{REFERENCES}

1. European Resuscitation Council Guidelines for Basic and Advanced Life Support (1992). Resuscitation 24(2), 103-123.

2. American College of Surgeons Committee on Trauma (1992) Advanced Trauma Life Support.

3. Dawson D.L., Johnson K.H. \& Jurkovich G.J. (1991) Course for Physicians American College of Surgeons Chicago. Injuries to the portal triad. American Journa of Surgery 161(5), 545-551.

4. Henne-Bruns D., Kremer D., Lloyd D.M. \& MeyerPannwitt U. (1933) Injuries of the portal vein in patients with blunt abdominal trauma. World Journal of Hepatic Pancreatic and Biliary Surgery 6(3), 163-168. 